Research Article

\title{
Infinitely Many Weak Solutions of the $p$-Laplacian Equation with Nonlinear Boundary Conditions
}

\author{
Feng-Yun Lu ${ }^{1,2}$ and Gui-Qian Deng ${ }^{1}$ \\ ${ }^{1}$ Xingyi Normal University for Nationalities, Xingyi, Guizhou 562400, China \\ ${ }^{2}$ Human Resources and Social Security Bureau, Buyi and Miao Autonomous Prefecture in Southwest Guizhou, Guizhou 562400, China \\ Correspondence should be addressed to Feng-Yun Lu; lufengyun@gmail.com
}

Received 24 August 2013; Accepted 27 October 2013; Published 14 January 2014

Academic Editors: E. A. Abdel-Salam and P. Candito

Copyright (c) 2014 F.-Y. Lu and G.-Q. Deng. This is an open access article distributed under the Creative Commons Attribution License, which permits unrestricted use, distribution, and reproduction in any medium, provided the original work is properly cited.

We study the following $p$-Laplacian equation with nonlinear boundary conditions: $-\Delta_{p} u+\mu(x)|u|^{p-2} u=f(x, u)+g(x, u), x \in$ $\Omega,|\nabla u|^{p-2} \partial u / \partial n=\eta|u|^{p-2} u$, and $x \in \partial \Omega$, where $\Omega$ is a bounded domain in $\mathbb{R}^{N}$ with smooth boundary $\partial \Omega$. We prove that the equation has infinitely many weak solutions by using the variant fountain theorem due to Zou (2001) and $f, g$ do not need to satisfy the $(P . S)$ or $\left(P . S^{*}\right)$ condition.

\section{Introduction}

In this paper, we study the following $p$-Laplacian equation:

$$
\begin{gathered}
-\Delta_{p} u+\mu(x)|u|^{p-2} u=f(x, u)+g(x, u), \quad x \in \Omega, \\
|\nabla u|^{p-2} \frac{\partial u}{\partial n}=\eta|u|^{p-2} u, \quad x \in \partial \Omega,
\end{gathered}
$$

where $\Omega$ is a bounded domain in $\mathbb{R}^{N}$ with smooth boundary $\partial \Omega$ and $\partial / \partial n$ is the outer normal derivative, $-\Delta_{p} u=\operatorname{div}\left(|\nabla u|^{p-2} \nabla u\right)$ is the $p$-Laplacian with $1<p<N$, $\eta$ is real parameter, and

$$
\mu(x) \in L^{\infty}(\Omega) \text { satisfying ess } \inf _{x \in \bar{\Omega}} \mu(x)>0 .
$$

The perturbation functions $f, g$ satisfy the following conditions:

(F1) $f, g \in C(\bar{\Omega} \times \mathbb{R}, \mathbb{R})$ are odd in $u$;

(F2) there exist $\sigma, \delta \in(1, p), c_{1}>0, c_{2}>0, c_{3}>0$ such that

$$
c_{1}|u|^{\sigma} \leq f(x, u) u \leq c_{2}|u|^{\sigma}+c_{3}|u|^{\delta},
$$

for a.e. $x \in \Omega$ and $u \in \mathbb{R}$.
(F3) There exists $p<q<p^{*}$ (where $p^{*}=p N / N-p$ ) such that $|g(x, u)| \leq c\left(1+|u|^{q}\right)$ for a.e. $x \in \Omega$ and $u \in$ $\mathbb{R}$. Moreover, $\lim _{u \rightarrow 0} g(x, u) /|u|^{p-1}=0$ uniformly for $x \in \Omega$.

(F4) Assume that one of the following conditions hold:

(1) $\lim _{|u| \rightarrow \infty} g(x, u) /|u|^{p-2} u=0$ uniformly for $x \in$ $\Omega$;

(2) $\lim _{|u| \rightarrow \infty} g(x, u) /|u|^{p-2} u=-\infty$ uniformly for $x \in \Omega$; furthermore, $f(x, u) /|u|^{p-2} u$ and $g(x, u) /|u|^{p-2} u$ are decreasing in $u$ for $u$ is large enough;

(3) $\lim _{|u| \rightarrow \infty} g(x, u) /|u|^{p-2} u=\infty$ uniformly for $x \in \Omega ; g(x, u) /|u|^{p-2} u$ is increasing in $u$ for $u$ is large enough; moreover, there exists $\alpha>$ $\max \{\sigma, \delta\}$ such that

$\liminf _{|u| \rightarrow \infty} \frac{g(x, u) u-p G(x, u)}{|u|^{\alpha}} \geq c>0 \quad$ uniformly for $x \in \Omega$,

where $G(x, u)=\int_{0}^{u} g(x, t) d t$.

Remark 1. The above conditions were given in Zou [1] for the semilinear case $p=2$. 
Remark 2. A simple example which satisfies (F1)-(F4) is

$$
f(x, u)+g(x, u)=\mu|u|^{r-2} u+\gamma|u|^{s-2} u,
$$

where $1<r<p<s<p^{*}$.

Equation (1) is posed in the framework of the Sobolev space

$$
W^{1, p}(\Omega)=\left\{u \in L^{p}(\Omega): \int_{\Omega}|\nabla u|^{p} d x<\infty\right\},
$$

with the norm

$$
\|u\|=\left(\int_{\Omega}\left(|\nabla u|^{p}+\mu(x)|u|^{p}\right) d x\right)^{1 / p} .
$$

The corresponding energy functional of (1) is defined by

$$
\begin{aligned}
\Phi(u)= & \frac{1}{p} \int_{\Omega}\left(|\nabla u|^{p}+\mu(x)|u|^{p}\right) d x-\int_{\Omega} F(x, u) d x \\
& -\int_{\Omega} G(x, u) d x-\frac{\eta}{p} \int_{\partial \Omega}|u|^{p} d s,
\end{aligned}
$$

for $u \in W^{1, p}(\Omega)$, where $F(x, u)=\int_{0}^{u} f(x, t) d t$ and $d s$ is the measure on the boundary. It is easy to see that $\Phi \in$ $C^{1}\left(W^{1, p}(\Omega), \mathbb{R}\right)$ and

$$
\begin{aligned}
& \left\langle\Phi^{\prime}(u), v\right\rangle \\
& =\int_{\Omega}\left(|\nabla u|^{p-2} \nabla u \nabla v+\mu(x)|u|^{p-2} u v\right) d x \\
& \quad-\int_{\Omega} f(x, u) v d x-\int_{\Omega} g(x, u) v d x-\eta \int_{\partial \Omega}|u|^{p-2} u v d s,
\end{aligned}
$$

for all $u, v \in W^{1, p}(\Omega)$. It is well-known that the weak solution of (1) corresponds to the critical point of the energy functional $\Phi$ on $W^{1, p}(\Omega)$.

Remark 3. Under condition (2), it is easy to check that norm (7) is equivalent to the usual one, that is, the norm defined in (7) with $\mu(x) \equiv 1$.

In [2], the author researched $(1)(\eta=0)$ and obtained the existence of infinitely many weak solutions. Moreover, the existence of three solutions for (1) $(\eta=0, p>N)$ was researched in [3] by using a three-critical-point theorem due to Ricceri [4]. Also, some authors researched and obtained the existence of infinitely many weak solution without requiring any symmetric conditions and also with discontinuous nonlinearities; see $[5,6]$. Recently, this equation was studied by J.-H. Zhao and P.-H. Zhao [7] via Bartsch's dual fountain theorem in [8] and obtained the existence of infinitely many weak solutions for (1) under the case of Remark 2. They obtained the following theorem.

Theorem A. Let $f(x, t)+g(x, t)=\mu|u|^{r-2} u+\gamma|u|^{s-2} u$, where $1<r<p<s<p^{*}$. Then there exists a constant $\Lambda>$ 0 such that, for any $\eta<\Lambda$,
(1) for any $\gamma>, \mu \in \mathbb{R}$, (1) has a sequence of solutions $u_{k}$ such that $\Phi\left(u_{k}\right) \rightarrow \infty$ as $k \rightarrow \infty$;

(2) for any $\mu>0, \gamma \in \mathbb{R}$, (1) has a sequence of solutions $v_{k}$ such that $\Phi\left(v_{k}\right) \rightarrow 0^{-}$as $k \rightarrow \infty$.

The main ingredient for the proof of the above theorem is a dual fountain theorem in [8]. It should be noted that the $(P . S)$ or $\left(P . S^{*}\right)$ condition and its variants play an important role in this theorem and its application. While the variant fountain theorem in Zou [1] does not need not the (P.S) or $\left(P . S^{*}\right)$ condition, we obtain the following generalized result by using Zou's theorem.

Theorem 4. Assume that (F1)-(F4) hold; then there exists a constant $\Lambda>0$ such that, for any $\eta<\Lambda$, (1) has infinitely many weak solutions $\left\{u_{k}\right\}$ satisfying

$$
\Phi\left(u_{k}\right) \longrightarrow 0^{-} \text {as } k \longrightarrow \infty
$$

This paper is organized as follows. In Section 2, we recall some preliminary theorems and lemmas. In Section 3, we give the proof of Theorem 4 .

\section{Preliminaries}

In what follows, we make use of the following notations: $E$ (or $W^{1, p}(\Omega)$ ) denotes Banach space with the norm $\|\cdot\| ; E^{*}$ denotes the conjugate space for $E ; L^{p}(\Omega)$ denotes Lebesgue space with the norm $|\cdot|_{p} ;\langle\cdot, \cdot\rangle$ is the dual pairing of the spaces $E^{*}$ and $E$; we denote by $\rightarrow$ (resp., $\rightarrow$ ) the strong (resp., weak) convergence; $c, c_{1}, c_{2}, \ldots$ denote (possibly different) positive constants.

For completeness, we first recall the variant fountain theorem in Zou [1]. Let $E$ be a Banach space with norm $\|\cdot\|$ and $E=\overline{\oplus_{j \in N} X_{j}}$ with $\operatorname{dim} X_{j}<\infty$ for any $j \in N$. Set $Y_{k}=$ $\oplus_{j=0}^{k} X_{j}, Z_{k}=\overline{\oplus_{j=k}^{\infty} X_{j}}$.

Theorem 5 (see [1, Theorem 2.2]). The $C^{1}$-functional $\Phi_{\lambda}$ : $E \rightarrow \mathbb{R}$ defined by $\Phi_{\lambda}(u)=A(u)-\lambda B(u), \lambda \in[1,2]$, satisfies

(A1) $\Phi_{\lambda}$ maps bounded sets to bounded sets uniformly for $\lambda \in[1,2]$; furthermore, $\Phi_{\lambda}(-u)=\Phi_{\lambda}(u)$ for all $(\lambda, u) \in[1,2] \times E$.

(A2) $B(u) \geq 0$ for all $u \in E ; B(u) \rightarrow \infty$ as $\|u\| \rightarrow \infty$ on any finite dimensional subspace of $E$.

(A3) There exists $\rho_{k}>r_{k}>0$ such that

$$
a_{k}(\lambda):=\inf _{u \in Z_{k},\|u\|=\rho_{k}} \Phi_{\lambda}(u) \geq 0>b_{k}(\lambda):=\max _{u \in Y_{k},\|u\|=r_{k}} \Phi_{\lambda}(u) ;
$$

for all $\lambda \in[1,2]$,

$$
\begin{aligned}
d_{k}(\lambda) & :=\inf _{u \in Z_{k},\|u\| \leq \rho_{k}} \Phi_{\lambda}(u) \longrightarrow 0 \\
\text { as } k & \longrightarrow \infty \text { uniformly for } \lambda \in[1,2] .
\end{aligned}
$$


Then there exist $\lambda_{n} \rightarrow 1, u\left(\lambda_{n}\right) \in Y_{n}$, such that

$$
\begin{array}{r}
\left.\Phi_{\lambda_{n}}^{\prime}\right|_{Y_{n}}\left(u\left(\lambda_{n}\right)\right)=0, \quad \Phi_{\lambda_{n}}\left(u\left(\lambda_{n}\right)\right) \longrightarrow c_{k} \in\left[d_{k}(2), b_{k}(1)\right] \\
\text { as } n \longrightarrow \infty .
\end{array}
$$

Particularly, if $\left\{u\left(\lambda_{n}\right)\right\}$ has a convergent subsequence for every $k$, then $\Phi_{1}$ has infinitely many nontrivial critical points $\left\{u_{k}\right\} \in E \backslash\{0\}$ satisfying $\Phi_{1}\left(u_{k}\right) \rightarrow 0^{-}$as $k \rightarrow \infty$.

Remark 6. Obviously, the sequence $\left\{u\left(\lambda_{n}\right)\right\}$ is a $\left(P . S^{*}\right)$ sequence.

For our working space $E=W^{1, p}(\Omega), E$ is a reflexive and separable Banach space; then there are $e_{j} \in E$ and $e_{j}^{*} \in E^{*}$ such that

$$
\begin{gathered}
E=\overline{\operatorname{span}\left\{e_{j}: j=1,2, \ldots\right\},} \quad E^{*}=\overline{\operatorname{span}\left\{e_{j}^{*}: j=1,2, \ldots\right\}}, \\
\left\langle e_{j}^{*}, e_{j}\right\rangle= \begin{cases}1, & i=j, \\
0, & i \neq j .\end{cases}
\end{gathered}
$$

We write $X_{j}:=\operatorname{span}\left\{e_{j}\right\}$; then $Y_{k}, Z_{k}$ can be defined as that in the beginning of Theorem 5 . Consider $\Phi_{\lambda}: E \rightarrow \mathbb{R}$ defined by

$$
\begin{aligned}
\Phi_{\lambda}(u):= & \frac{1}{p}\|u\|^{p}-\int_{\Omega} G(x, u) d x-\frac{\eta}{p} \int_{\partial \Omega}|u|^{p} d s \\
& -\lambda \int_{\Omega} F(x, u) d x \\
:= & A(u)-\lambda B(u), \quad \lambda \in[1,2] .
\end{aligned}
$$

Then $B(u) \geq 0$ for all $u \in E ; B(u) \rightarrow \infty$ as $\|u\| \rightarrow \infty$ on any finite dimensional subspace of $E ; \Phi_{\lambda}(-u)=\Phi_{\lambda}(u)$ for all $\lambda \in[1,2], u \in E$. We need the following lemmas.

Lemma 7 (see [7, Lemma 3.5]). If $1 \leq q<p^{*}$, then one has

$$
\beta_{k}:=\sup _{u \in Z_{k},\|u\|=1}|u|_{q} \longrightarrow 0 \text { as } k \longrightarrow \infty \text {. }
$$

\section{Proof of Theorem 4}

First, we check the condition of Theorem 5.

Lemma 8. Assume (F1)-(F3); then (A1)-(A3) hold.

Proof. (A1) and (A2) are obvious. Let $n>k>2$; we assume that $\sigma \leq \delta$ and define

$$
\beta_{k}(\sigma):=\sup _{u \in Z_{k},\|u\|=1}|u|_{\sigma}, \quad \beta_{k}(\delta):=\sup _{u \in Z_{k},\|u\|=1}|u|_{\delta} .
$$

Observe that

$$
|u|_{\sigma} \leq \beta_{k}(\sigma)\|u\|, \quad|u|_{\delta} \leq \beta_{k}(\delta)\|u\|,
$$

for any $u \in Z_{k}$. Note that $q<p^{*}$; there exists a constant $c>0$ such that

$$
|u|_{q} \leq c\|u\| .
$$

By the Sobolev trace imbedding inequality, we have

$$
|u|_{L^{p}(\partial \Omega)}^{p} \leq K\|u\|^{p} .
$$

Then we take $\Lambda^{*}=1 / 4 K$ such that, for all $\eta<\Lambda^{*}$,

$$
\frac{\eta}{p}|u|_{L^{p}(\partial \Omega)}^{p} \leq \frac{1}{4 p}\|u\|^{p} .
$$

By (F3), for any $\varepsilon>0$, there exists $c_{\varepsilon}$ such that

$$
|G(x, u)| \leq \varepsilon|u|^{p}+c_{\varepsilon}|u|^{q} .
$$

Then, by (F1)-(F3) and (18)-(21), we obtain

$$
\begin{aligned}
\Phi_{\lambda}(u)= & \frac{1}{p}\|u\|^{p}-\int_{\Omega} G(x, u) d x-\frac{\eta}{p} \int_{\partial \Omega}|u|^{p} d s \\
& -\lambda \int_{\Omega} F(x, u) d x \\
\geq & \frac{3}{4 p}\|u\|^{p}-\varepsilon|u|_{p}^{p}-c_{\varepsilon}|u|_{q}^{q}-c|u|_{\sigma}^{\sigma}-c|u|_{\delta}^{\delta} \\
\geq & \frac{3}{4 p}\|u\|^{p}-\varepsilon c\|u\|^{p}-c\|u\|^{q}-c \beta_{k}(\sigma)^{\sigma}\|u\|^{\sigma} \\
& -c \beta_{k}(\delta)^{\delta}\|u\|^{\delta} .
\end{aligned}
$$

Note that $p<q$; we may choose $\varepsilon>0$ and $R>0$ sufficiently small that

$$
\frac{1}{4 p}\|u\|^{p}-\varepsilon c\|u\|^{p}-c\|u\|^{q} \geq 0
$$

holds true for any $u \in W^{1, p}(\Omega)$ with $\|u\| \leq R$. So we have

$$
\Phi_{\lambda}(u) \geq \frac{1}{2 p}\|u\|^{p}-c \beta_{k}(\sigma)^{\sigma}\|u\|^{\sigma}-c \beta_{k}(\delta)^{\delta}\|u\|^{\delta},
$$

for any $u \in Z_{k}$ with $\|u\| \leq R$. Choosing

$$
\rho_{k}:=\left(4 p c \beta_{k}(\sigma)^{\sigma}+4 p c \beta_{k}(\delta)^{\delta}\right)^{1 /(p-\delta)},
$$

by Lemma 7 , for $\beta_{k}(\sigma) \rightarrow 0, \beta_{k}(\delta) \rightarrow 0$ as $k \rightarrow \infty$, it follows that $\rho_{k} \rightarrow 0$ as $k \rightarrow \infty$, so there exists $k_{0}$ such that $\rho_{k} \leq R$ when $k \geq k_{0}$. Thus, for $k \geq k_{0}, u \in Z_{k}$, and $\|u\|=\rho_{k}$, we have $\Phi_{\lambda}(u) \geq \rho_{k}^{p} / 4 p>0$; then $a_{k}(\lambda) \geq 0$ for all $\lambda \in[1,2]$.

On the other hand, if $u \in Y_{k}$ with $\|u\|$ being small enough, since all the norms are equivalent on the finite dimensional space and $\sigma<p$, then $b_{k}(\lambda)<0$ for all $\lambda \in[1,2]$.

Furthermore, if $u \in Z_{k}$ with $\|u\| \leq \rho_{k}, k \geq k_{0}$, we see that

$$
\Phi_{\lambda}(u) \geq-c \beta_{k}(\sigma)^{\sigma} \rho_{k}^{\sigma}-c \beta_{k}(\delta)^{\delta} \rho_{k}^{\delta} \longrightarrow 0 \quad \text { as } k \longrightarrow \infty .
$$

Therefore, $d_{k}(\lambda) \rightarrow 0$ as $k \rightarrow \infty$. Thus, (A3) holds.

By Theorem 5, we have the following lemma. 
Lemma 9. There exist $\lambda_{n} \rightarrow 1$ and $u\left(\lambda_{n}\right) \in Y_{n}$ such that

$$
\begin{aligned}
& \Phi_{\left.\lambda_{n}\right|_{n}}^{\prime}\left(u\left(\lambda_{n}\right)\right)=0, \\
& \Phi_{\lambda_{n}}\left(u\left(\lambda_{n}\right)\right) \longrightarrow c_{k} \in\left[d_{k}(2), b_{k}(1)\right] \text { as } n \longrightarrow \infty .
\end{aligned}
$$

In order to complete our proof of Theorem 4 , by a standard argument (see the proof of Lemma 3.4 in Zhao [7]), we only need to show that $\left\{u\left(\lambda_{n}\right)\right\}$ is bounded.

Lemma 10. $\left\{u\left(\lambda_{n}\right)\right\}$ is bounded in $W^{1, p}(\Omega)$.

Proof. Since $\left.\Phi_{\lambda_{n}}^{\prime}\right|_{Y_{n}}\left(u\left(\lambda_{n}\right)\right)=0$, then

$$
\begin{aligned}
1 & -\eta \int_{\partial \Omega} \frac{\left|u\left(\lambda_{n}\right)\right|^{p}}{\left\|u\left(\lambda_{n}\right)\right\|^{p}} d s \\
& =\int_{\Omega} \frac{\lambda_{n} f\left(x, u\left(\lambda_{n}\right) u\left(\lambda_{n}\right)\right)+g\left(x, u\left(\lambda_{n}\right)\right) u\left(\lambda_{n}\right)}{\left\|u\left(\lambda_{n}\right)\right\|^{p}} d x .
\end{aligned}
$$

We can choose $0<\Lambda<\Lambda^{*}$ and if $\eta<\Lambda$ such that $1-\eta K>0$. If, up to a subsequence, $\left\|u\left(\lambda_{n}\right)\right\| \rightarrow \infty$ as $n \rightarrow \infty$, then, by (F2),

$$
1+|\eta| K \geq \int_{\Omega} \frac{g\left(x, u\left(\lambda_{n}\right)\right) u\left(\lambda_{n}\right)}{\left\|u\left(\lambda_{n}\right)\right\|^{p}} d x \geq \frac{1}{2}(1-\eta K),
$$

for $n$ is large enough. Obviously, it is a condition if (F4)(1) holds.

Otherwise, we set $w_{n}=u\left(\lambda_{n}\right) /\left\|u\left(\lambda_{n}\right)\right\|$; then, up to a subsequence,

$$
\begin{gathered}
w_{n} \rightarrow w \text { in } E, \\
w_{n} \longrightarrow w \text { in } L^{t}(\Omega) \quad \text { for } 1 \leq t<p^{*}, \\
w_{n}(x) \longrightarrow w(x) \quad \text { a.e. } x \in \Omega .
\end{gathered}
$$

If $w \neq 0$ in $E$ and $\lim _{|u| \rightarrow \infty} g(x, u) /|u|^{p-2} u=-\infty$ in (F4)(2), then, for $n$ is large enough, by Fatou's Lemma, we have that

$$
\begin{aligned}
& -\frac{1}{2}(1-\eta K) \\
& \quad \geq \int_{\Omega} \frac{-g\left(x, u\left(\lambda_{n}\right)\right) u\left(\lambda_{n}\right)}{\left|u\left(\lambda_{n}\right)\right|^{p}}\left|w_{n}\right|^{p} d x \\
& \quad \geq c+\int_{\left\{x \in \Omega: w \neq 0,\left|u\left(\lambda_{n}\right)\right| \geq c\right\}} \frac{-g\left(x, u\left(\lambda_{n}\right)\right) u\left(\lambda_{n}\right)}{\left|u\left(\lambda_{n}\right)\right|^{p}}\left|w_{n}\right|^{p} d x \\
& \quad \longrightarrow \infty ;
\end{aligned}
$$

this is a contradiction. It is similar if $\lim _{|u| \rightarrow \infty} g(x, u) /|u|^{p-2} u=\infty$ in (F4)(3). Thus, $w=0$.

Let $t_{n} \in[0,1]$ such that

$$
\Phi_{\lambda_{n}}\left(t_{n} u\left(\lambda_{n}\right)\right):=\max _{t \in[0,1]} \Phi_{\lambda_{n}}\left(t u\left(\lambda_{n}\right)\right) .
$$

For any $c>0$ large enough, and $\bar{w}_{n}:=(2 p c)^{1 / p} w_{n}$, for $n$ is large enough, we have that

$$
\begin{aligned}
\Phi_{\lambda_{n}}\left(t_{n} u\left(\lambda_{n}\right)\right) \geq & \Phi_{\lambda_{n}}\left(\bar{w}_{n}\right) \\
= & 2 c-\int_{\Omega} G\left(x, \bar{w}_{n}\right) d x-\frac{\eta}{p} \int_{\partial \Omega}\left|\bar{w}_{n}\right|^{p} d s \\
& -\lambda_{n} \int_{\Omega} F\left(x, \bar{w}_{n}\right) d x
\end{aligned}
$$$$
\geq c \text {, }
$$

which implies that $\lim _{n \rightarrow \infty} \Phi_{\lambda_{n}}\left(t_{n} u\left(\lambda_{n}\right)\right) \rightarrow \infty$. Obviously,

$$
\left\langle\Phi_{\lambda_{n}}^{\prime}\left(t_{n} u\left(\lambda_{n}\right)\right), t_{n} u\left(\lambda_{n}\right)\right\rangle=0
$$

It follows that

$$
\begin{gathered}
\infty=\lim _{n \rightarrow \infty}\left(\Phi_{\lambda_{n}}\left(t_{n} u\left(\lambda_{n}\right)\right)-\frac{1}{p}\left\langle\Phi_{\lambda_{n}}^{\prime}\left(t_{n} u\left(\lambda_{n}\right)\right), t_{n} u\left(\lambda_{n}\right)\right\rangle\right) \\
\leq \lim _{n \rightarrow \infty} \lambda_{n} \int_{\Omega}\left(\frac{1}{p} f\left(x, t_{n} u\left(\lambda_{n}\right)\right) t_{n} u\left(\lambda_{n}\right)\right. \\
\left.-F\left(x, t_{n} u\left(\lambda_{n}\right)\right)\right) d x \\
+\int_{\Omega}\left(\frac{1}{p} g\left(x, t_{n} u\left(\lambda_{n}\right)\right) t_{n} u\left(\lambda_{n}\right)-G\left(x, t_{n} u\left(\lambda_{n}\right)\right)\right) d x .
\end{gathered}
$$

If (F4)(2) holds, we have that $(1 / p) f(x, u) u-F(x, u)$ and $(1 / p) g(x, u) u-G(x, u)$ are decreasing in $u$ for $u$ is large enough. Therefore,

$$
\frac{1}{p} f(x, s u) s u-F(x, s u)+\frac{1}{p} g(x, s u) s u-G(x, s u) \leq c
$$

for all $s>0$ and $u \in \mathbb{R}$; it is a contradiction.

If (F4)(3) holds, then we have that

$$
\begin{aligned}
\infty \leq c & \int_{\Omega}\left|u\left(\lambda_{n}\right)\right|^{\sigma} d x \\
& +\int_{\Omega}\left(\frac{1}{p} g\left(x, u\left(\lambda_{n}\right)\right) u\left(\lambda_{n}\right)-G\left(x, u\left(\lambda_{n}\right)\right)\right) d x,
\end{aligned}
$$

which implies

$$
\int_{\Omega}\left(\frac{1}{p} g\left(x, u\left(\lambda_{n}\right)\right) u\left(\lambda_{n}\right)-G\left(x, u\left(\lambda_{n}\right)\right)\right) d x \longrightarrow \infty .
$$


On the other hand, by the property of $u\left(\lambda_{n}\right)$, for $n$ is large enough, since $\alpha>\max \{\delta, \sigma\}$, we have that

$$
\begin{aligned}
b_{k}(1) & \lambda_{n} \int_{\Omega}\left(\frac{1}{p} f\left(x, u\left(\lambda_{n}\right)\right) u\left(\lambda_{n}\right)-F\left(x, u\left(\lambda_{n}\right)\right)\right) d x \\
& +\int_{\Omega}\left(\frac{1}{p} g\left(x, u\left(\lambda_{n}\right)\right) u\left(\lambda_{n}\right)-G\left(x, u\left(\lambda_{n}\right)\right)\right) d x \\
\geq & \frac{1}{2} \int_{\Omega}\left(\frac{1}{p} g\left(x, u\left(\lambda_{n}\right)\right) u\left(\lambda_{n}\right)-G\left(x, u\left(\lambda_{n}\right)\right)\right) d x \\
& +\frac{1}{2} c \int_{\Omega}\left|u\left(\lambda_{n}\right)\right|^{\alpha} d x-\frac{1}{2} c \int_{\Omega}\left|u\left(\lambda_{n}\right)\right|^{\delta} d x \\
& -\frac{1}{2} c \int_{\Omega}\left|u\left(\lambda_{n}\right)\right|^{\sigma} d x \\
\geq & c \int_{\Omega}\left(\frac{1}{p} g\left(x, u\left(\lambda_{n}\right)\right) u\left(\lambda_{n}\right)-G\left(x, u\left(\lambda_{n}\right)\right)\right) d x-c ;
\end{aligned}
$$

this implies that $\int_{\Omega}\left((1 / p) g\left(x, u\left(\lambda_{n}\right)\right) u\left(\lambda_{n}\right)-G\left(x, u\left(\lambda_{n}\right)\right)\right) d x$ is bounded, which contradicts (39).

By the above arguments, we have that $\left\{u\left(\lambda_{n}\right)\right\}$ is bounded.

Remark 11. In fact, our result still holds if we consider a weaker condition than (F4)(2); that is, there is $c>0$ such that

$$
H(x, t) \leq H(x, s)+c, \quad \bar{H}(x, t) \leq \bar{H}(x, s)+c
$$

for all $0<s<t$ or $t<s<0, \forall x \in \Omega$, where $H(x, t)=$ $(1 / p) f(x, t) t-F(x, t)$ and $\bar{H}(x, t)=(1 / p) g(x, t) t-G(x, t)$.

\section{Conflict of Interests}

The authors declare that there is no conflict of interests regarding the publication of this paper.

\section{References}

[1] W.-M. Zou, "Variant fountain theorems and their applications," Manuscripta Mathematica, vol. 104, no. 3, pp. 343-358, 2001.

[2] B. Ricceri, "Infinitely many solutions of the Neumann problem for elliptic equations involving the $p$-Laplacian," Bulletin of the London Mathematical Society, vol. 33, no. 3, pp. 331-340, 2001.

[3] G. Bonanno and P. Candito, "Three solutions to a Neumann problem for elliptic equations involving the $p$-Laplacian," Archiv der Mathematik, vol. 80, no. 4, pp. 424-429, 2003.

[4] B. Ricceri, "On a three critical points theorem," Archiv der Mathematik, vol. 75, no. 3, pp. 220-226, 2000.

[5] D. Giuseppina and S. Angela, "Infinitely many solutions to elliptic problems with variable exponent and nonhomogeneous Neumann conditions," Nonlinear Analysis: Theory, Methods \& Applications, vol. 75, pp. 5612-5619, 2012.

[6] P. Candito, "Infinitely many solutions to the Neumann problem for elliptic equations involving the $p$-Laplacian and with discontinuous nonlinearities," Proceedings of the Edinburgh Mathematical Society, vol. 45, no. 2, pp. 397-409, 2002.

[7] J.-H. Zhao and P.-H. Zhao, "Existence of infinitely many weak solutions for the $p$-Laplacian with nonlinear boundary conditions," Nonlinear Analysis, Theory, Methods and Applications, vol. 69, no. 4, pp. 1343-1355, 2008.

[8] T. Bartsch and M. Willem, "On an elliptic equation with concave and convex nonlinearities," Proceedings of the American Mathematical Society, vol. 123, pp. 3555-3561, 1995. 


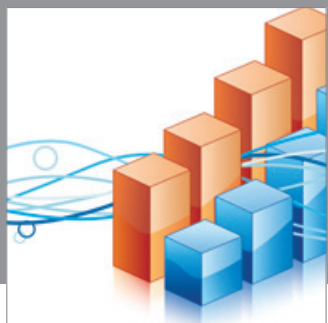

Advances in

Operations Research

mansans

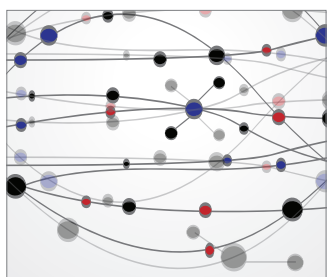

The Scientific World Journal
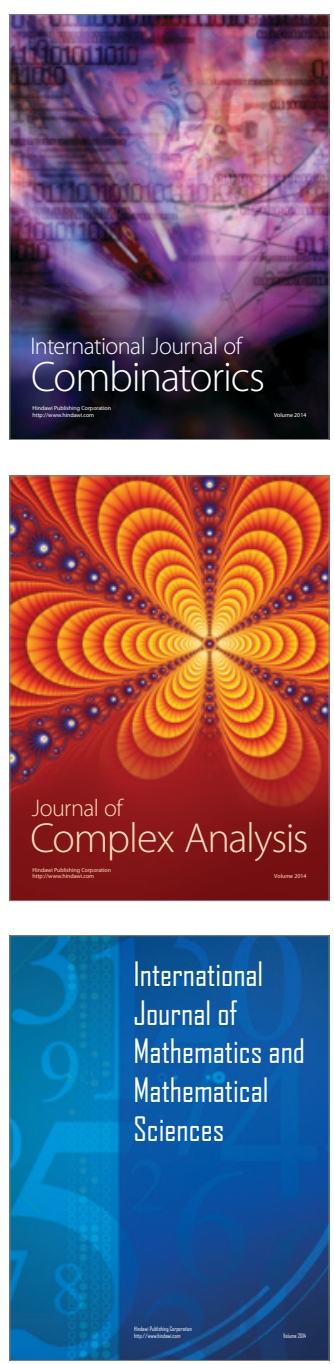
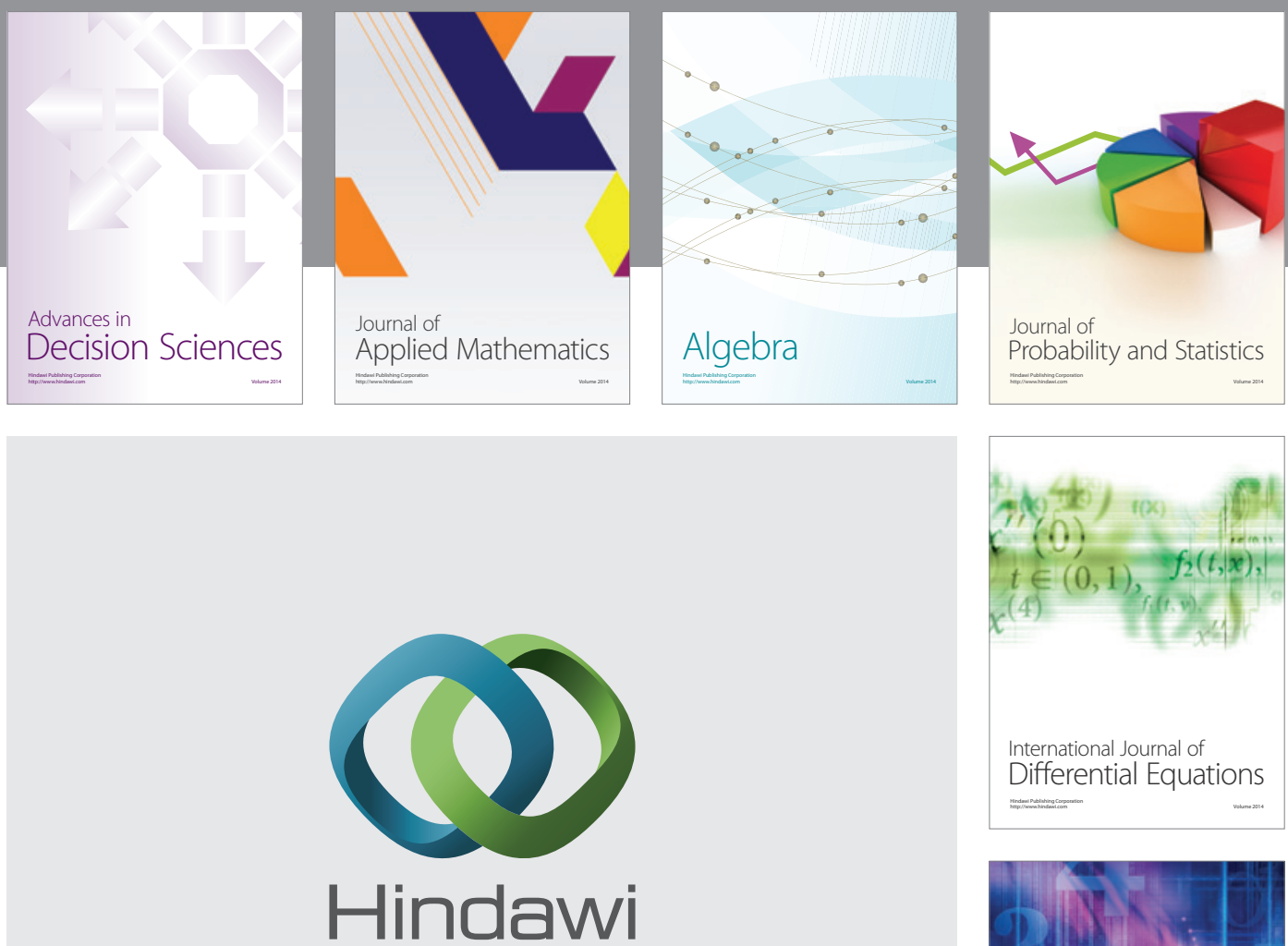

Submit your manuscripts at http://www.hindawi.com
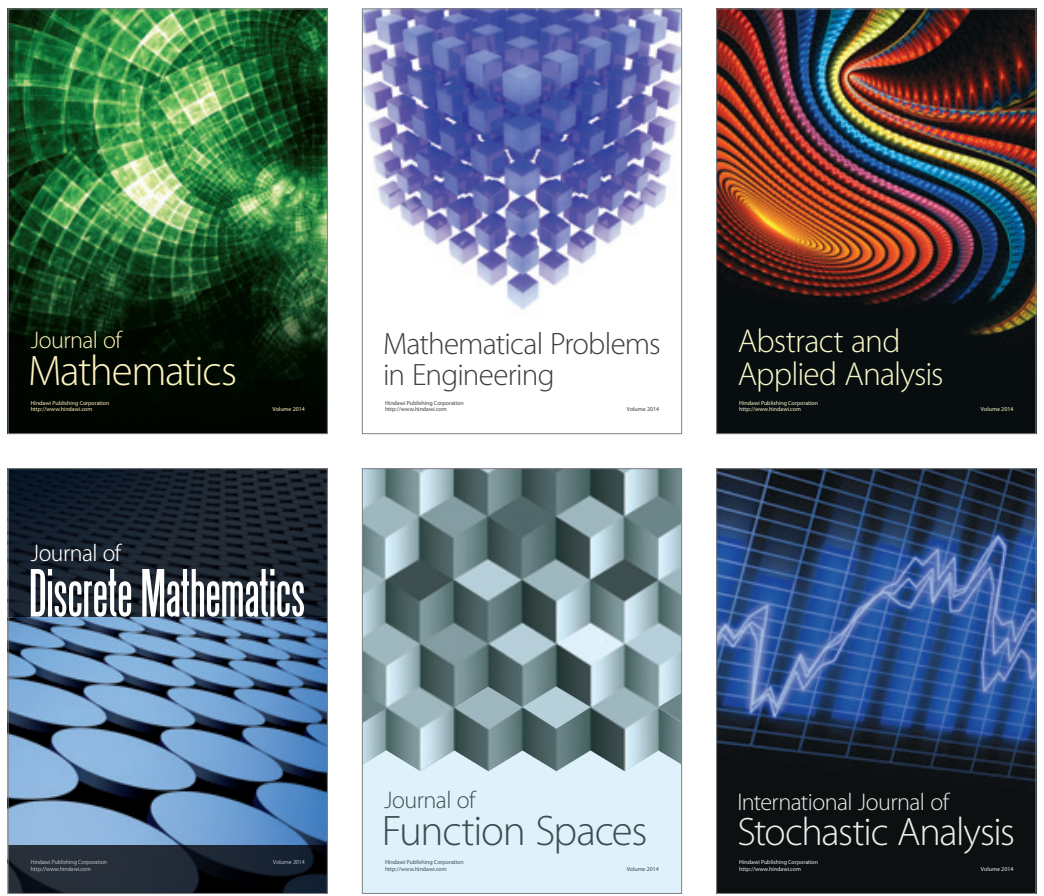

Journal of

Function Spaces

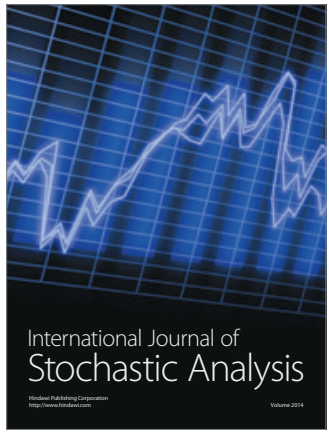

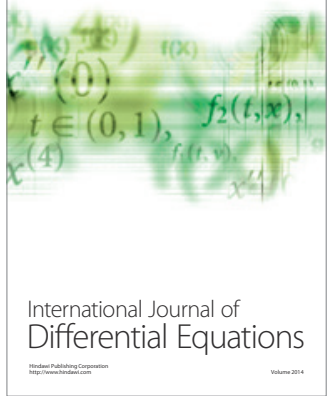
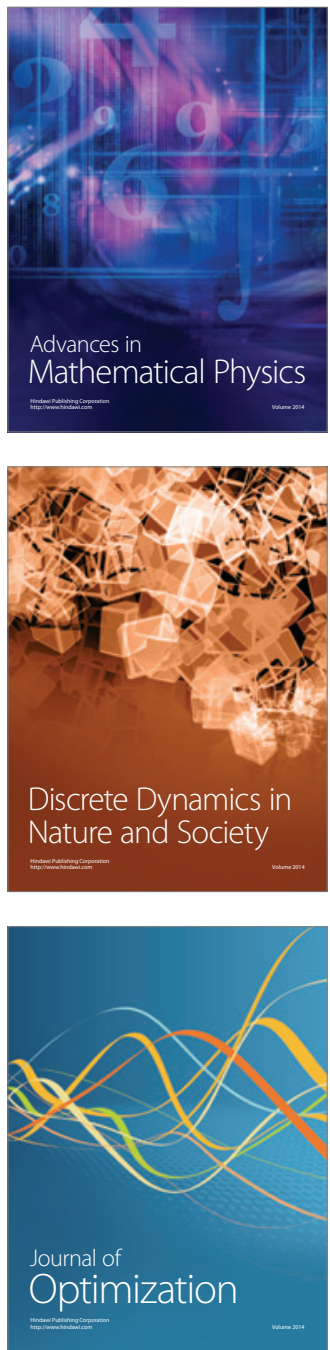